\section{WIRE-MESH CAPACITANCE TOMOGRAPHY FOR TREATMENT PLANNING SYSTEM OF ELECTRO- CAPACITIVE CANCER THERAPY}

Anis Nismayantia,b*, Marlin R. Baidillahd, Triwikantorob, Endarkob*, Warsito P. Tarunoc,d

aPhysics Department, University of Tadulako, Palu 94118, Central Sulawesi, Indonesia

bPhysics Department, Institut Teknologi Sepuluh Nopember, Kampus ITS Sukolilo - Surabaya 60111 , East Java, Indonesia

cPhysics Department, University of Indonesia, Depok 16424, Indonesia

'Center for Medical Physics and Cancer Research C-Tech Labs

Edwar Technology, Tangerang 15325, Indonesia
Article history

Received

31 October 2019

Received in revised form

27 July 2021

Accepted

4 August 2021

Published online 20 October 2021

\section{Graphical abstract}

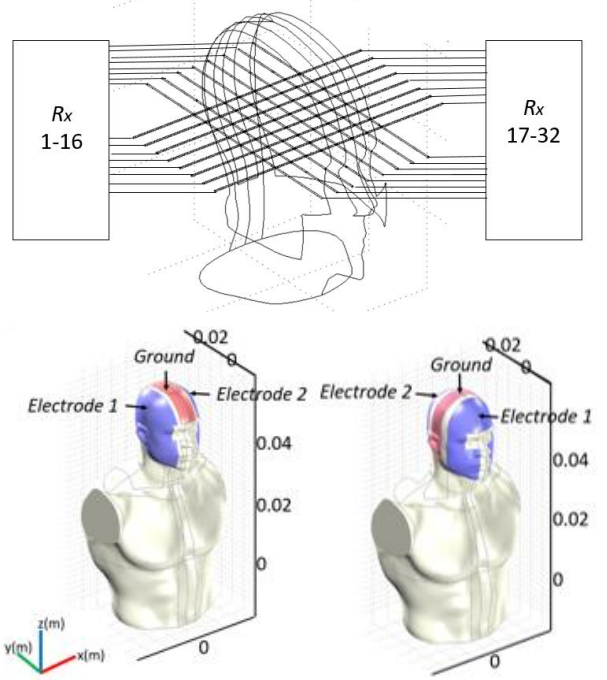

\begin{abstract}
The wire-mesh capacitance tomography (WMCT) has been applied to visualize $2 \mathrm{D}$ of the distribution of electric field intensity in the treatment planning system (TPS) of electro-capacitive cancer therapy (ECCT) using human head model. WMCT is proposed in this study to estimate accurately the distribution of electric field intensity which is the main optimum factors of ECCT in order to compensate the inaccuracy of TPS ECCT simulation. The experimental and simulation studies were conducted with wire-mesh sensor consisted of $8 \times 8$ wire matrix of copper in human head model using two type of helmet ECCT. The result of electric field value at the intersection wire-mesh have been compared between experimental studies and simulation studies. The electric field average value resulted from ECCT helmet-1 is higher than ECCT helmet-2. The average electric field generated by the ECCT helmet-1 is $1585.72 \mathrm{~V} / \mathrm{m}$ in an air medium, $97.43 \mathrm{~V} / \mathrm{m}$ in grey matter and $80.58 \mathrm{~V} / \mathrm{m}$ in the cancer. While the average electric field generated by the ECCT helmet-2 is $1413.28 \mathrm{~V} / \mathrm{m}$ in an air medium, $64.20 \mathrm{~V} / \mathrm{m}$ in grey matter and $52.65 \mathrm{~V} / \mathrm{m}$ in the cancer. ECCT helmet-1 and helmet-2 result the different of electric field distribution pattern. ECCT helmet-1 is more optimal for used to patient has cancer position in the right and bottom, while ECCT helmet-2 is more optimal for used to patient has cancer position in the top and bottom. Based on this study, the compensation error of the electrical field distribution of simulation study can be applied in order to enhance the accuracy of TPS ECCT. Therefore, it envisages that this study paves way the development of better approaches for general electric field therapy.
\end{abstract}

Keywords: Electro-capacitive cancer therapy (ECCT), wire-mesh capacitive tomography (WMCT), treatment planning system (TPS), electric field intensity distribution 


\subsection{INTRODUCTION}

Malignant brain and central nervous system (CNS) tumor among persons age 0-14 years were the most common cause of cancer death [1]. The cancer treatment method that utilizes intermediate-frequency and low intensity electric fields placed on the skin surface is new breakthrough in the development of cancer treatment technology $[2,3,4,5]$. The antiproliferative effect of low intensity and intermediate frequency electrostatic was investigated in vitro and in vivo and showed the breast tumor cells were reduced significant of more than $67 \%$ in size [6]. The exposure electric field provided divides cancer cells causes an increment of tubulin A, which affects the polymerization of microtubules to be inhibited. Disruption of microtubule polymerization will prevent the cell division from progressing to the anaphase stage. The anaphase stage can be prevented by interfering with the dynamics of microtubules using an electric field. So that dividing cancer cells is disrupted by the division process and causes cancer cell death [7].

Electro-capacitive cancer therapy (ECCT) is the proposed methods which uses the alternating electric field for brain cancer treatment $[7,8]$. ECCT consists of capacitive electrode clothes which cover the surface of the body. This capacitive technique uses a quasistatic electric field produced by a voltage generator. The design of the 3D ECCT electrode is very important to treatment planning system (TPS) of ECCT to inhibit the tumor area in the human body. A good ECCT design will produce an optimal intensity electric field in the tumor area. The intensity electric field whose penetrate the body in the form of nonlinear causes an optimal intensity electric field to be difficult to achieve. Simulations intensity of the proper electric field intensity distribution in the 3D biomaterial phantom have been carried out [9]. But simulation need to be validated. So that measurement is needed to get the intensity of electric field distribution to increase the accuracy of the ECCT TPS.

Wire-mesh sensors are already used to visualize the permittivity distribution and the conductivity in industrial processes from bubbling fluid flow $[10,11,12,13]$. This wire-mesh sensor is very effective for locate void fraction in the crossing of wire. So wire-mesh sensors are an option for measure the distribution of electric field intensity as a compensation error for inaccurate ECCT TPS numerical simulations. Recently, effect of the wiremesh on the electric field intensity distribution generated by the ECCT system for helmet on the dielectric medium has been simulated [14]. And our previous study about the experiments to validate the simulation results have done for only one cancer location and a helmet type of ECCT by using wire-mesh tomography [15]. The standard method to evaluate of this ECCT system as a part of TPS as well as other electrotherapy modalities is by using a numerical simulation. The accuracy of numerical simulation studies is limited to evaluate the interfacial polarization between two biological tissue layers that affecting the electric distribution and the anisotropic medium. Because modelling this interfacial polarization and anisotropic phenomenon require high cost computation.

In this study we investigated the cancers of five different location, and we compare two type of helmet ECCT to analyze the distribution of electric field intensity of ECCT based on the 3D numerical simulation and experimental studies in human head model.

\subsection{METHODOLOGY}

Wire-Mesh Capacitance Tomography for ECCT on Human Head Model

In ECCT TPS, the design of capacitive electrodes is important because every patient has a different case with the different cancer location. The different case must be treated with different electrode designs to get the most optimal electric field in the cancer. The ECCT system have several electrodes of capacitive and is connected to generator AC voltage via coaxial cable [8]. The capacitive electrodes is attached to particular human body such as human head and breast. This capacitive electrode acts as two parallel plates to interfere the growth of cancer cells. The generator AC voltage produces voltage polarity alternating to electrode with $\mathrm{Vac}=+10 \mathrm{~V}$ or $-10 \mathrm{~V}$ and frequency $f=$ 100 - $300 \mathrm{kHz}$. The voltage source comes from a rechargeable battery with a maximum current charging of $\mathrm{I}=0.35 \mathrm{~A}$.

The capacitive electrodes ECCT in the human head model consists of two helmet type of capacitive electrodes as shown in Figure 1. It only becomes as a transmitter for both electrodes since the wires in WMCT is as the receivers. Helmet-1 have capacitive electrodes position at sagittal plane which are at the left and right side. Helmet-1 includes ground electrode at the middle between capacitive electrodes. The different configuration of helmet-2 is capacitive electrodes position at coronal plane are at the front and back. Helmet-2 also includes ground electrode at the middle between capacitive electrodes.

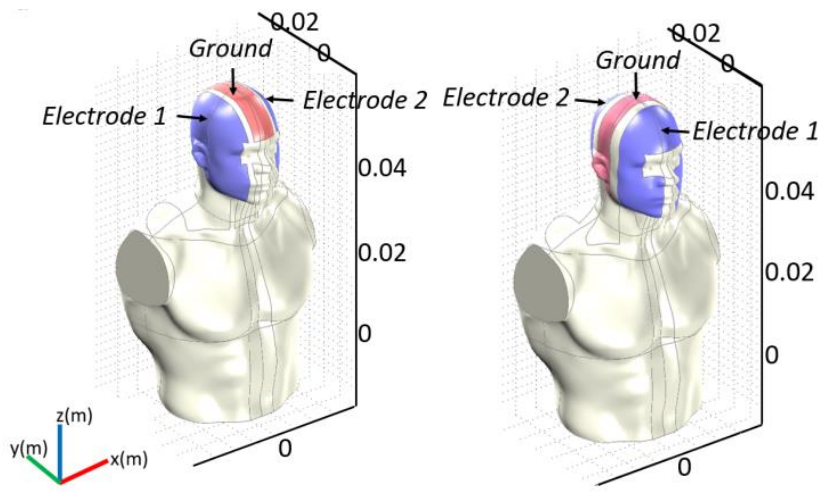

(a)

(b)

Figure 1 The model of 3D human head: (a) helmet-1, (b) helmet-2 


\section{WMCT Sensor for Human Head Model}

The WMCT is employed to visualize the electric field intensity distribution at each crossing point of wire. The WMCT sensor is placed in human head model on the axial axis to measure the electric field intensity distribution. Wire material is copper because it has a high conductivity value so it is good for measuring the distribution of electric field intensity. There are other materials that can be used such as silver and gold but copper is recommended in this study. Figure 2 shows the schematic of data acquisition for WMCT sensor in the case of a human head model.

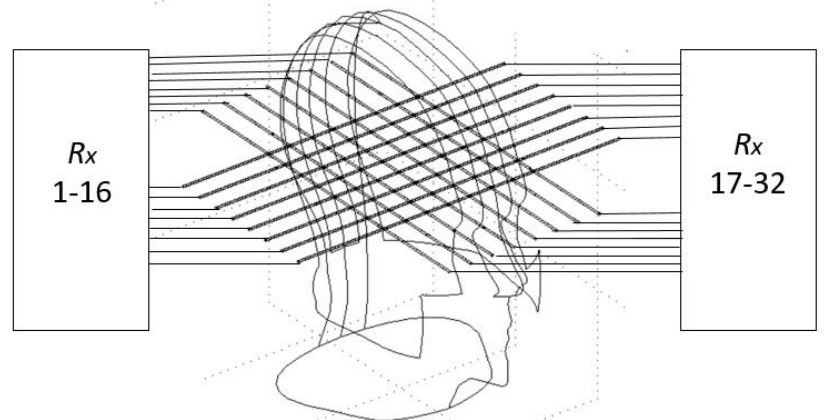

Figure 2 Schematic of WMCT sensor on human head model

The WMCT sensor consist of $8 \times 8$ mesh matrix intersect each other arranged in parallel with $90^{\circ}$ angles (see Figure 3(a)). The dimensions are $I=0.14 \mathrm{~m}$ in length, $D=0.001 \mathrm{~m}$ in diameter, and $r=0.01 \mathrm{~m}$ in distance of mesh. On this study, WMCT sensor behave only as receiver wires $R_{x}$ without being as a transmitter wires $T_{x}$. From $8 \times 8$ mesh count, it has $p=64$ point of the wire crossing with the number of receiver wire-end $n=$ 32. $R_{x} 1$ till 32 produces a different value at the end of detection point on the same wire, because the voltage source comes from capacitive electrodes ECCT with two type of helmet in different configurations. In order to avoid the alteration electric field intensity distribution of ECCT, thus the transmitter wire $T_{x}$ is avoided.

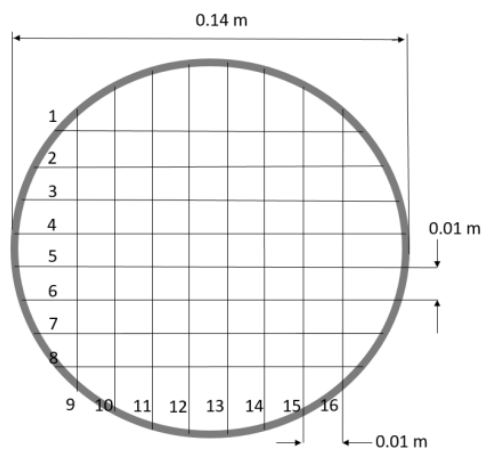

(a)

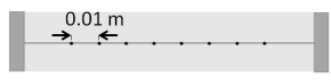

(b)
Figure 3 The schematic of WMCT sensor (a) top view, (b) side view
Image Reconstruction of WMCT

Meanwhile, in order to increase the sensitivity of detection electric field distribution, the perpendicular wire-mesh is connected as shown in Figure 3(b) [14]. This wire-mesh sensor configuration differs as compared with the conventional wire-mesh sensor which has a small gap distance between the perpendicular wire [12]. In order to reconstruct the electric field intensity distribution from the series of voltage measurements, we employed the sensitivitybased image reconstruction that is linear back projection [16] because of a single pair voltage measurement represents the voltage value from the $p$ $=64$ points of the wire crossings. In this regards, the conventional WMCT image reconstruction such as calibrated data image, binary image processing algorithms [17], cannot be used in this study.

The idea to predict the electric field distribution $\mathbf{E}$ is by firstly predict the electric flux $d \Phi$ at wire crossing. According to Gauss's law, the electric flux $d \Phi\left(\sim d Q / \varepsilon_{0}\right)$ through an area $\mathrm{d} \mathbf{A}$ is the electric field $\mathbf{E}$ multiplied by the surface area projected in a plane perpendicular to the field $\mathrm{d} \mathbf{A}$. In terms of discretization in a matrix of each point wire crossing $p$, it can be written as follows:

$$
E_{p}=\frac{\mathrm{d} \Phi}{\mathrm{d} A_{p}}=\frac{\mathrm{d} Q_{p}}{\varepsilon_{0} \mathrm{~d} A_{p}}
$$

Where, $E_{p}[\mathrm{~V} / \mathrm{m}], \mathrm{d} \Phi\left[\mathrm{C} / \mathrm{m}^{2}\right], \mathrm{d} A_{p}\left[\mathrm{~m}^{2}\right]$ are the intensity of electric field in $p$, the electric flux in $p$, and the area of point wire crossing $p$ respectively. $\varepsilon_{0}$ is the permittivity vacuum. Due to the nonlinear relationship between the measured capacitance data $C_{i j}$ or the accumulated charge $Q_{p}$ with the relative permittivity $\varepsilon_{\text {rp }}$ at point wire crossing $p$, the Frechét derivative is used to make the linearization [18, 19] in equation [2] and then Jacobian matrix $S$ in equation [4] can be used as a main part for image reconstruction. Due to its computational cost, it is quite commonly used due to its faster other than method such as rank-one update method.

$$
\delta Q_{p}=\delta \varepsilon_{r p} \frac{\mathrm{d} Q_{p}}{\mathrm{~d} \varepsilon_{r p}}=\delta \varepsilon_{r p}\left(S_{n}\right)_{p}
$$

The discretization of relative permittivity at point wire crossing $\delta \varepsilon_{r p}$ can be described from the reconstructed permittivity distribution $G$.

$$
G=\sum_{p=1}^{p=64} \varepsilon_{r p} \chi_{p}
$$

where $\chi_{p}$ is one in the point wire crossing and zero otherwise. While in equation [4], it is regarded a Jacobian matrix that mathematically form of as follows: 


$$
S_{(i j) p}=\frac{\mathrm{d} Q_{(i j) p}}{\mathrm{~d} \varepsilon_{p}}=\int \chi_{p} \nabla \phi_{i} \cdot \nabla \phi_{j} \mathrm{~d} x \mathrm{~d} y \mathrm{~d} z
$$

where $S_{(i j)}$ is a Jacobian matrix between wire-end $i$-th and ECCT electrode $j$-th. $\phi_{i}$ is a potential distribution when the wire-end $i$-th is defined as a voltage receiver. While, $\phi_{j}$ is a potential distribution when the ECCT electrode $j$-th is defined as a voltage source. In equation [2], the Jacobian matrix $S$ with dimension $[p x$ $n]$ is normalized into $S_{n}$ with dimension $[p \times 1]$ :

$$
\left(S_{n}\right)_{p}=\frac{S_{p}}{\sum_{i=1}^{i=32} S_{(i j) p}}
$$

\section{Simulation Setup}

Simulation of the electric field intensity distribution in a human head model was carried out based on the finite element method (FEM) using COMSOL Multiphysics with AC/DC module [20]. This simulation calculates the electric field distribution with a given alternating voltage by the capacitive electrodes as shown in Figure 1. Domain computation on a conductive medium is appropriate model that match with experimental study.

The human head model use relative permittivity of grey matter $\varepsilon_{r-g}=3221.8$ and relative permittivity of cancer $\varepsilon_{r-c}=5000$. The relative permittivity values of grey matter and cancer follows the cited references [21]. The dimensions of the head are $I=0.17 \mathrm{~m}$ in length, $w=0.16 \mathrm{~m}$ in width and $h=0.11 \mathrm{~m}$ in height for grey matter as shown in Figure 4(a). The dimension of the cancer is $r=0.002 \mathrm{~m}$ in radius as shown in Figure $4(\mathrm{~b})$. Furthermore, on the anatomy of the head given cancer with five different locations are in the middle, right, left, top and bottom (see Figure 5).

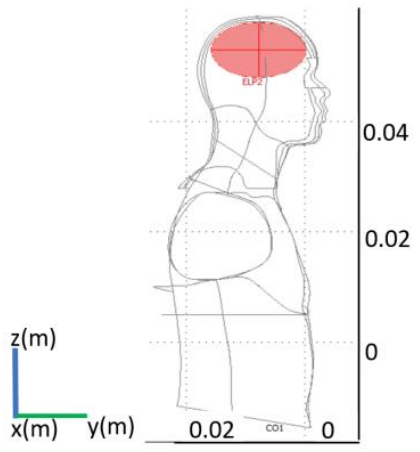

(a)

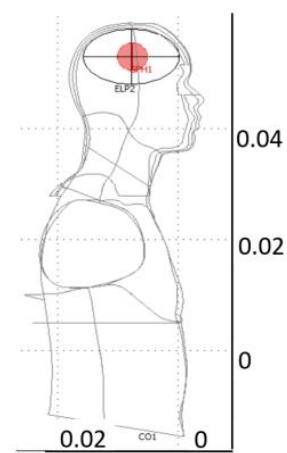

(b)
Figure 4 The side view of human head model with: (a) grey matter, (b) cancer

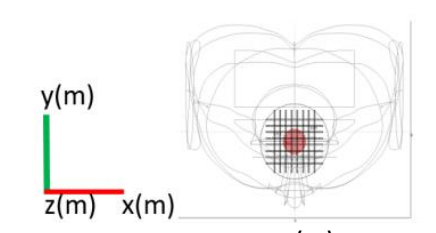

(a)

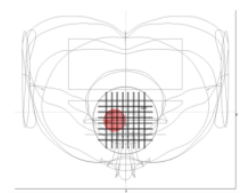

(c)

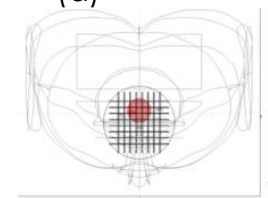

(d)

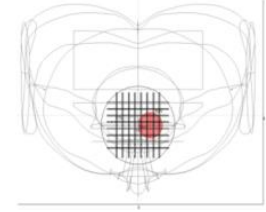

(b)

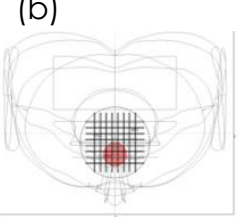

(e)
Figure 5 Cancer location in the human head model from the top view: (a) middle, (b) right, (c) left, (d) top (e) bottom

\section{Experimental Setup}

An experimental study on human head model is shown in Figure 6. Voltage of capacitive electrodes $V_{p}$ $p=10$ Volt, and frequency $f=100 \mathrm{kHz}$. The biomaterial phantom represents as the grey matter was made from the mixture of $\mathrm{H}_{2} \mathrm{O} 41.3 \%$, Glycerin $24.8 \%$, Silicon rubber $24.8 \%$, Agar $4.5 \%$ and $\mathrm{NaCl} 0.5 \%$ as shown in Figure 6(a). Meanwhile, the cancer was made from the mixture of $\mathrm{H}_{2} \mathrm{O} 41.3 \%$, Glycerin $24.8 \%$, Silicon rubber $24.8 \%$, Agar $4.5 \%$ and $\mathrm{NaCl} 2.5 \%$ as shown in Figure 6(b).

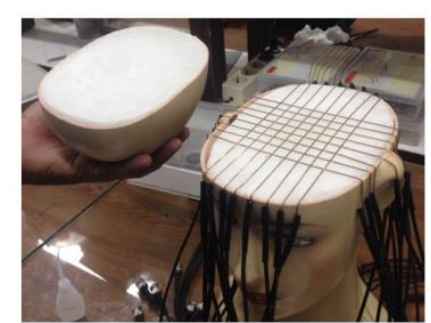

(a)

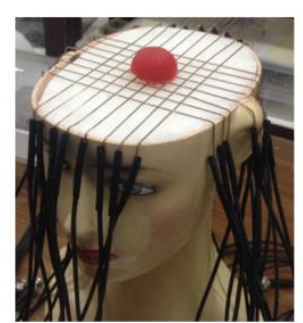

(b)
Figure 6 (a) Human head model with biomaterial phantom, and (b) human head model with biomaterial phantom and cancer

\subsection{RESULTS AND DISCUSSION}

The result of simulation electric field distribution generated by the ECCT helmet-1 and helmet-2 in air medium can be seen in Figure 7. Different electrode design between helmet -1 and helmet-2 give a different pattern of the electric field intensity distribution. 


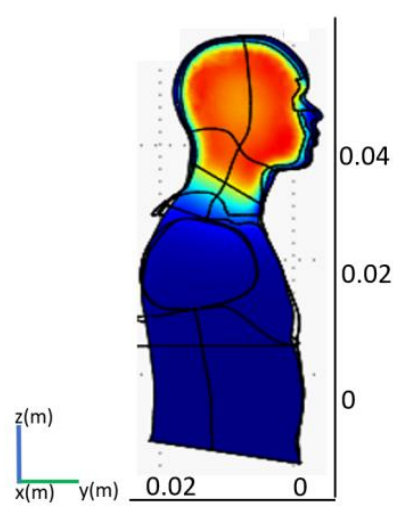

(a)

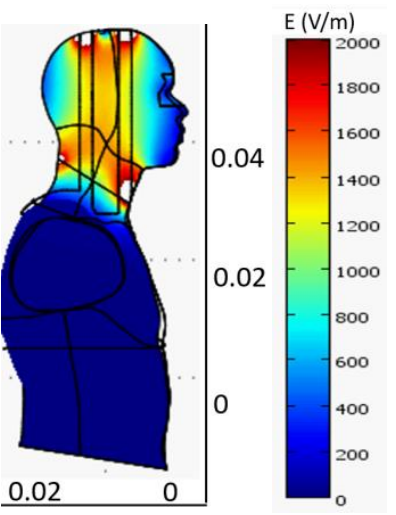

(b)
Figure 7 The simulation result of electric field intensity distribution with ECCT: (a) helmet-1, (b) helmet-2

Figure 7 shows electric field distribution in the human head model without wire-mesh sensor, with a red colour indicating the highest electric field and blue colour indicate lower electric field. ECCT helmet-1 results electric field distribution is highest as long as $y$ axis. While helmet-2 results electric field distribution is highest as long as $x$ axis.

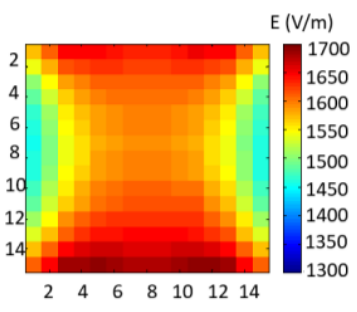

(a)

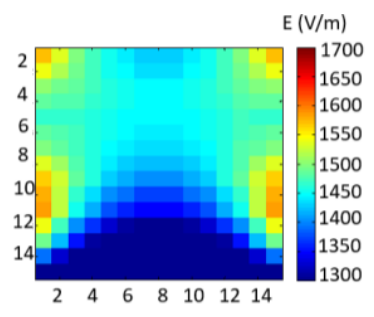

(b)
Figure 8 The simulation result of electric field intensity distribution by using WMCT in air medium with ECCT: (a) helmet-1, (b) helmet-2

Figure 7 and 8 shows electric field distribution generated by system ECCT on the human head model in the case of air medium. The average of electric field distribution generated by ECCT helmet- 1 on air medium is $1585.72 \mathrm{~V} / \mathrm{m}$, while ECCT helmet-2 is $1413.28 \mathrm{~V} / \mathrm{m}$. Figure 8 shows a different electric field distribution pattern between ECCT helmet-1 and helmet-2 in the form of tomography with wire-mesh. This occurs because different electrode design between helmet-1 and helmet-2 (can be seen in Figure 1). This different electrode design will make different of direction electric field. Alamsyah et al. [6] reported that increasing the number of field directions from the positions of the capacitive electrode pairs, resulted in potentially anti-proliferative effect of electrocapacitive cancer therapy in vivo (>67\%).

The experimental result of reconstruction electric field intensity distribution on the grey matter and cancer with ECCT helmet- 1 in Figure 9 is summarised in Table 1. The electric field average value are $97.43 \mathrm{~V} / \mathrm{m}$ in grey matter and $80.58 \mathrm{~V} / \mathrm{m}$ in cancer. The electric field in cancer lower than in grey matter, because the relative permittivity of cancer is greater than grey matter. The electric field distribution obtained at 64 point of the wire crossings represents a permittivity distribution. Due to the relationship between the electric field $\mathbf{E}$ and permittivity $\rho$ based on Gauss's Theory [22].

$$
\nabla \cdot \mathbf{E}=\frac{\rho}{\varepsilon_{0}}
$$

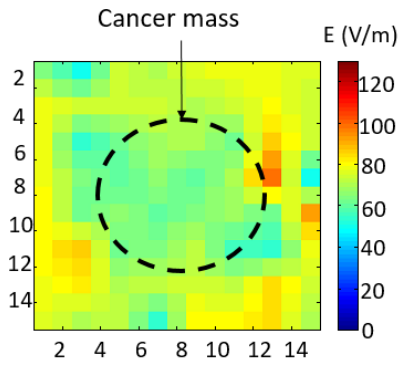

(a)

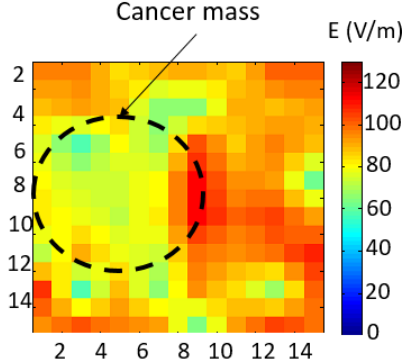

(c)

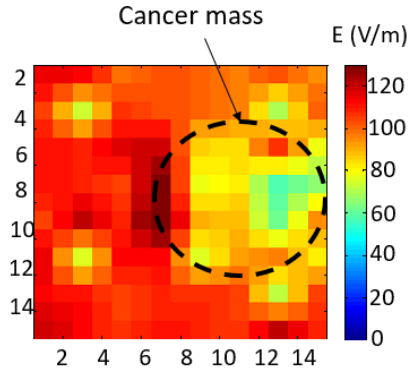

(b)

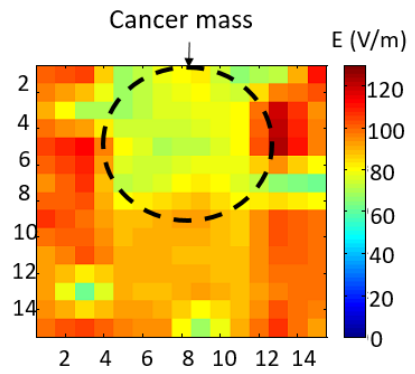

(d)

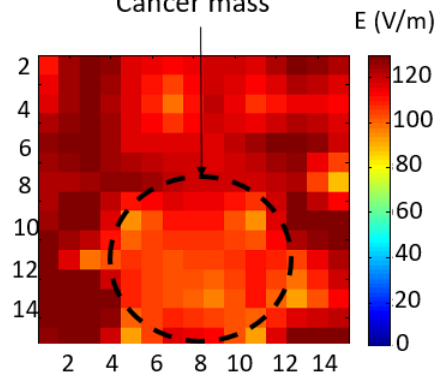

(e)

Figure 9 Reconstruction result of electric field distribution on human head with ECCT helmet-1 with five variation cancer position: (a) middle, (b) right, (c) left, (d) top, (e) bottom

Figure $9(a)$ shows electric field distribution generated by ECCT helmet-1 with cancer position in the middle. The electric field average value in this tomography are $73.82 \mathrm{~V} / \mathrm{m}$ in grey matter and 64.27 $\mathrm{V} / \mathrm{m}$ in the cancer. Figure $9(\mathrm{~b})$ shows electric field distribution generated by ECCT helmet-1 with cancer position in the right. The electric field average value in this tomography are $106.30 \mathrm{~V} / \mathrm{m}$ in grey matter and $82.60 \mathrm{~V} / \mathrm{m}$ in the cancer. 
Table 1 The electric field average value distribution

\begin{tabular}{clcccc}
\hline \multirow{2}{*}{ No. } & \multirow{2}{*}{$\begin{array}{l}\text { Cancer } \\
\text { position }\end{array}$} & \multicolumn{2}{c}{$\begin{array}{c}\text { ECCT helmet-1 (V/m) } \\
\text { matter }\end{array}$} & Cancer & \multicolumn{2}{c}{ ECCT helmet-2 (V/m) } \\
matter & Cancer \\
\hline 1. & Middle & 73.82 & 64.27 & 42.42 & 34.40 \\
2. & Right & 106.3 & 82.60 & 50.09 & 41.92 \\
3. & Left & 91.07 & 76.36 & 62.08 & 52.36 \\
4. & Top & 93.84 & 75.51 & 81.33 & 64.05 \\
5. & Bottom & 122.10 & 104.15 & 85.09 & 70.50 \\
\hline
\end{tabular}

The result of reconstruction electric field distribution on the grey matter and cancer with ECCT helmet-2 in Figure 10 is summarized in Table 1. The electric field average value are $64.20 \mathrm{~V} / \mathrm{m}$ in grey matter and 52.65 $\mathrm{V} / \mathrm{m}$ in cancer. The electric field in cancer lower than in grey matter. The same case with ECCT helmet-1.

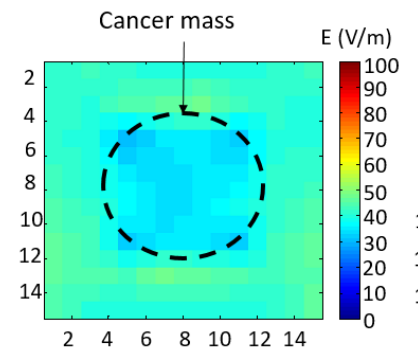

(a)

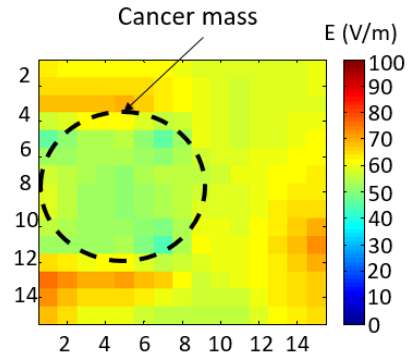

(c)

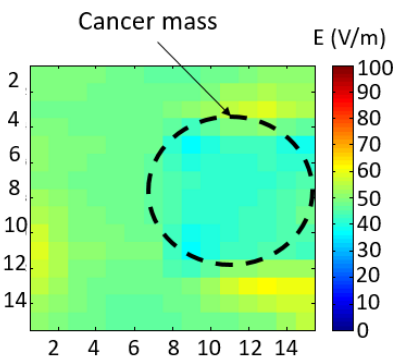

(b)

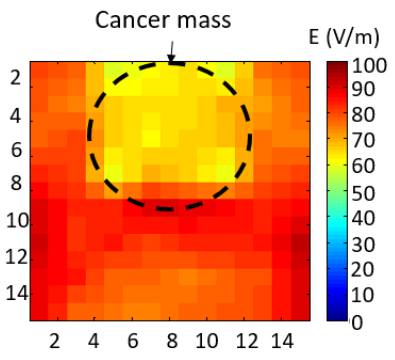

(d)

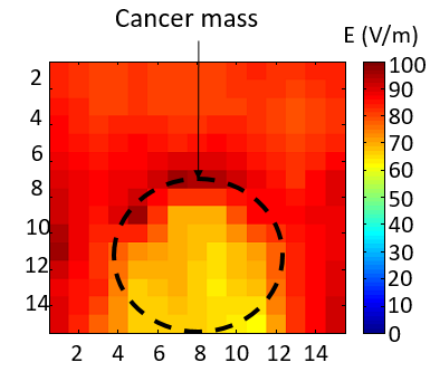

(e)

Figure 10 Reconstruction result of the electric field distribution on human head with ECCT helmet-2 with five variation cancer position: (a) middle, (b) right, (c) left, (d) top, (e) bottom

This result also in accordance with Andiani et al. research [14]. Andiani et al. reported that electric field in air medium is $173.48 \mathrm{~V} / \mathrm{m}$ greater than electric field in brain white matter is $0.46 \mathrm{~V} / \mathrm{m}$ and in a cancer is 0.17 $\mathrm{V} / \mathrm{m}$.
The compensation error in this study is used severity $\left(\mathrm{Sev}_{\mathrm{e}}\right)$ score as one of the error significance analysis [23]. This relates to the error source from the measured electric field. The result of compensation error for two type of ECCT is given in Table 2.

Table 2 Compensation Error

\begin{tabular}{clcccc}
\hline No. & $\begin{array}{l}\text { Cancer } \\
\text { position }\end{array}$ & $\begin{array}{l}\text { ECCT } \\
\text { helmet-1 (\%) }\end{array}$ & Sev $_{\text {ECCT }}$ & $\begin{array}{l}\text { EClmet-2 (\%) } \\
\text { helmet }\end{array}$ & S $_{\text {ev }}$ \\
\hline 1. & Middle & 27.64 & 5 & 1.30 & 1 \\
2. & Right & 48.09 & 5 & 15.07 & 5 \\
3. & Left & 40.89 & 5 & 31.73 & 5 \\
4. & Top & 40.40 & 5 & 47.80 & 5 \\
5. & Bottom & 55.31 & 10 & 49.98 & 5 \\
\hline
\end{tabular}

The electric field distribution at each intersection wire can be detected through simulations and experiments when given an external voltage source through the ECCT capacitive electrode pair. In the simulation, the electric field value at each intersection wire can be obtained directly. Whereas in the experiment, to get the electric field value at each intersection wire requires reconstruction. In experiments, each wire channel acts as a receiver to detect the electric field generated by ECCT. Then the reconstruction is conducted to get the electric field distribution at each intersection wire. The relationship between simulation results and actual results is shown in Table 2 compensation errors. The expectation is that the simulation results and experimental results have a small error.

ECCT helmet- 1 and helmet-2 result the different of electric field distribution pattern. ECCT helmet-1 is more optimal for used to patient has cancer position in the right and bottom, because the highest electric field is achieved in this cancer position. While ECCT helmet-2 is more optimal for used to patient has cancer position in the top and bottom. Because the highest electric field is achieved in this tumor position (see Table 1). The electric field average value from ECCT helmet-1 is higher than ECCT helmet-2. The advantage of this study is to become the basis of general electric field therapy in measure electric field distribution.

\subsection{CONCLUSION}

The wire-mesh capacitance tomography (WMCT) has been applied to predict the quantification of the electric field distribution in human head model for treatment planning system of ECCT. The result of electric field value at the intersection wire-mesh have been compared between experimental studies and simulation studies. The electric field average value resulted from ECCT helmet-1 is higher than ECCT helmet-2. The average electric field generated by the ECCT helmet-1 is $1585.72 \mathrm{~V} / \mathrm{m}$ in an air medium, 97.43 $\mathrm{V} / \mathrm{m}$ in grey matter and $80.58 \mathrm{~V} / \mathrm{m}$ in the cancer. While the average electric field generated by the ECCT 
helmet-2 is $1413.28 \mathrm{~V} / \mathrm{m}$ in an air medium, $64.20 \mathrm{~V} / \mathrm{m}$ in grey matter and $52.65 \mathrm{~V} / \mathrm{m}$ in the cancer. ECCT helmet-1 and helmet-2 result the different of electric field distribution pattern. ECCT helmet-1 is more optimal for used to patient has cancer position in the right and bottom, while ECCT helmet-2 is more optimal for used to patient has cancer position in the top and bottom.

\section{Acknowledgement}

All authors have contributed equally to this work. This work was supported in part by the C-Tech Lab Edwar Technology and in part by Directorate General of Higher Education, DIKTI, Indonesia.

\section{References}

[1] Q. T. Ostrom, H. Gittleman, G. Truitt, A. Boscia, C. Kruchko, and J. S. Barnholz-Sloan. 2018. CBTRUS Statistical Report: Primary Brain and Other Central Nervous System Tumors Diagnosed in the United states in 2011-2015. Neuro Oncol. 1:20(suppl_4): iv1-iv86.

[2] E. D. Kirson et al. 2004. Disruption of Cancer Cell Replication by Alternating Electric Fields. Cancer Research. 64(9): 328895.

[3] E. D. Kirson et al. 2007. Alternating Electric Fields Arrest Cell Proliferation in Animal Tumor Models and Human Brain Tumors. Proceedings of the National Academy of Sciences. 104(24): 10152-10157.

[4] M. Salzberg. 2008. A Pilot Study with Very Low-intensity, Intermediate-frequency Electric Fields in Patients with Locally Advanced and/or Metastatic Solid Tumors. Onkologie. 31: 362-365.

[5] S. G. Turner, T. Gergel, H. Wu, M. Lacroix, and S. A. Toms. 2014. The Effect of Field Strength on Glioblastoma Multiforme Response in Patients Treated with the NovoTTF TM -100A System. World Journal of Surgical Oncology. 12: 1-5.

[6] F. Alamsyah, I. N. Ajrina, F. N. A. Dewi, D. Iskandriati, S. A. Prabandari, and W. P. Taruno. 2017. Antiproliferative Effect of Electric Fields on Breast Tumor Cells in Vitro and In Vivo. Indonesian Journal of Cancer Chemoprevention. 6(3): 71.

[7] Sahudi, F. Alamsyah, and Warsito. 2017. Cell Death and Induced p53 Expression in Oral Cancer, HeLa, and Bone Marrow Mesenchyme Cells under the Exposure to Noncontact Electric Fields. Integrative Medicine International. 4: 161-170.

[8] Warsito. 2016. Metode Kompensasi Kapasitansi Parasitik pada Tomografi Listrik. Hak Paten HAKI P 00201600087.

[9] M. Giladi et al. 2014. Alternating Electric Fields (TumorTreating Fields Therapy) Can Improve Chemotherapy Treatment Efficacy in Non-Small Cell Lung Cancer Both in Vitro and in Vivo. Seminars in Oncology. 41: S35-S41.

[10] H. M. Prasser, D. Scholz, and C. Zippe. 2001. Bubble Size Measurement using Wire-mesh Sensors. Flow Measurement and Instrumentation. 12: 299-312.

[11] B. J. Azzopardi et al. 2010. Comparison between Electrical Capacitance Tomography and Wire Mesh Sensor Output for Air/Silicone Oil Flow in a Vertical Pipe. Industrial \& Engineering Chemistry Research. 49(18): 8805-8811.

[12] H. F. Velasco Pena and O. M. H. Rodriguez. 2015. Applications of Wire-mesh Sensors in Multiphase Flows. Flow Measurement and Instrumentation. 45: 255-273.

[13] C. Y. Ofuchi et al. 2019. Multiple Wire-mesh Sensors Applied to the Characterization of Two-Phase Flow inside a Cyclonic Flow Distribution System †. Sensors. 19(193): 1-13,

[14] L. Andiani, Endarko, M. Al Huda, and Warsito. 2017. A Novel Method for Analyzing Electric Field Distribution of Electro Capacitive Cancer Treatment (ECCT) Using Wire Mesh Electrodes: A Case Study of Brain Cancer Therapy. EuroMediterranean Biomedical Journal. 12(38): 178-183,

[15] A. Nismayanti, M. R. Baidillah, L. Andiani, T. Triwikantoro, E. Endarko, and W. P. Taruno. 2018. Electric Field Distribution Measurement for Electro-capacitive Cancer Therapy by using Wire Mesh Tomography. Procedings of iBioMed 2018. 49-52.

[16] W. Warsito, Q. Marashdeh, and L. S. Fan. 2007. Electrical Capacitance Volume Tomography. IEEE Sensors Journal. 7(4): 525-535

[17] M. J. Da Silva, E. Schleicher, and U. Hampel. 2007. Capacitance Wire-mesh Sensor for Fast Measurement of Phase Fraction Distributions. Measurement Science and Technology. 18(7): 2245-2251.

[18] W. R. B. Lionheart. 2001. Reconstruction Algorithms for Permittivity and Conductivity Imaging. Proc. of the 2nd World Congress on Industrial Process Tomography (Hannover, Germany, August 2001). 4-11.

[19] D. B. Geselowitz, 1971. An Application of Electrocardiographic Lead Theory to Impedance Plethysmography. IEEE Transactions on Biomedical Engineering. 18(1): 38-41.

[20] Comsol. 2017. Introduction to COMSOL Multiphysics 5.3.

[21] L. Thompson and P. R. Campos. 2013. A Head and Neck Simulator for Radiology and Radiotherapy. IEEE Transactions on Nuclear Science. 60(3): 1503-1511.

[22] D. H. Staelin. 2011. Electromagnetics and Applications. Department of Electrical Engineering and Computer Science Massachusetts Institute of Technology Cambridge, MA.

[23] S. Eastwood and P. Webb. 2009. Error Significance Analysis and Compensation for HPKMs. Industrial Robot: An International Journal. 36(1): 27-35. 